\title{
Factores asociados a la gestión de quejas contra médicos que laboran en hospitales del Instituto Mexicano del Seguro Social
}

\author{
Raúl Peña-Viveros, MC, ${ }^{(1)}$ José Raymundo Rodríguez-Moctezuma, MC , M en Invest Clin, ${ }^{(2)}$ \\ Juan Manuel López-Carmona, $\mathrm{M}$ en Invest Clin.(3)
}

Peña-Viveros R, Rodríguez-Moctezuma JR, López-Carmona JM.

Factores asociados a la gestión de quejas contra médicos que laboran en hospitales del Instituto Mexicano del Seguro Social. Salud Publica Mex 2004;46:210-215. El texto completo en inglés de este artículo está disponible en: http://www.insp.mx/salud/index.html

\section{Resumen}

Objetivo. Identificar los factores asociados a la gestión de quejas contra los médicos que trabajan en hospitales generales del Instituto Mexicano del Seguro Social. Material y métodos. Se revisaron las quejas presentadas y los expedientes clínicos correspondientes, en los nueve hospitales de la Delegación Estado de México O riente, del Instituto Mexicano del Seguro Social. Por cada expediente clínico con queja se seleccionaron como controles dos expedientes sin queja, de la misma unidad y servicio. Se buscaron asociaciones por medio de razón de momios y regresión logística. Resultados. Se incluyeron 130 expedientes motivo de queja y 260 expedientes control. Se identificaron siete factores asociados a queja de 14 investigados: complicaciones durante la atención RM 2.8 (IC 95\% 1.3-5.8), error diagnóstico RM 3.18 (IC 95\% 1.7-5.6), utilización de estudios de gabinete RM 3.7 (IC 95\% 1.2-11.3), información deficiente por parte del médico RM 2.64 (IC 95\% 1.2-5.7), alta voluntaria RM 7.2 (IC 95\% 2.2-23.6), falta de vigilancia durante la hospitalización RM 19.12 (IC 95\% 2.2-162.6) y revisiones vaginales múltiples durante el trabajo de parto RM 5.17 (IC 95\% 1.5-17.07). Hubo tendencia a la presentación de quejas cuando se reportaron: mala relación médico-paciente, deficiente atencion del trabajo de parto, error terapéutico y diferimiento de cirugía, sin significancia estadística. Conclusiones. Las quejas contra médicos de hospitales

\author{
Peña-Viveros R, Rodríguez-Moctezuma JR, \\ López-Carmona JM. \\ Factors associated with complaints \\ against physicians working at Mexican Institute \\ of Social Security hospitals. \\ Salud Publica Mex 2004;46:210-215. \\ The English version of this paper \\ is available at: http://www.insp.mx/salud/index.html
}

\begin{abstract}
A bstract
Objective.To identify the factors associated with complaints of patients against physicians who work at general hospitals of the Mexican Institute of Social Security (MISS). Material and Methods. All the medical complaint files in a three-year period at the nine general hospitals of Mexico State MISS East D istrict were examined. For each complaint filed, two control files were selected from the same hospital and clinical area. Associations were assessed using odds ratios and logistic regression. Results. A total of $130 \mathrm{com}$ plaint cases and 260 controls were included. Seven out of 14 risk factors were selected: complications during hospitalization (O R 2.8, 95\% Cl 1.3-5.8), diagnostic error (O R 3.18, $95 \% \mathrm{Cl} 1.7-5.6)$, use of diagnostic tests (O R $3.7,95 \% \mathrm{Cl}$ 1.2-11.3), insufficient information given by physicians (OR $2.64,95 \% \mathrm{CI} 1.22-5.7)$, voluntary hospital discharge (O R 7.2, $95 \% \mathrm{CI}$ 2.22-23.6), lack of clinical monitoring during hospitalization (O R 19.12, 95\% Cl 2.25-162.6), and multiple vaginal revisions during labor (OR $5.17,95 \% \mathrm{CI} 1.517 .07)$. Complaints were filed more often when there was a poor patient-physician relationship, deficient monitoring during labor, therapeutic error, and delay in surgery. Statistical significance was not attained. Conclusions. Complaints against Mexico State MISS hospital physicians were associated with diagnostic technical factors, as well as with the patients' perception of receiving deficient information and care dur-
\end{abstract}

(1) Dirección del Hospital General Regional 72 "Lic.Vicente Santos G uajardo". Tlalnepantla, Estado de México, México.

(2) Módulo de Investigación. Hospital General Regional 72 "Lic.Vicente Santos Guajardo". Tlalnepantla, Estado de México, México.

(3) Unidad de Medicina Familiar 91. Delegación Estado de México 0 riente. Instituto Mexicano del Seguro Social, Coacalco, Estado de México, México.

Fecha de recibido: 10 de junio de 2003 - Fecha de aprobado: 5 de abril de 2004

Solicitud de sobretiros: Dr. Raymundo Rodríguez Moctezuma. Hospital General Regional N 0. 72 "Lic.Vicente Santos Guajardo", Módulo de investigación, 2o piso, avenida Filiberto Gómez sin número, esquina Gustavo Baz, 54000 Tlalnepantla, Estado de México, México. Correo electrónico: rodmoc@ prodigy.net.mx 
del Instituto Mexicano del Seguro Social en el Estado de México se asocian con aspectos técnicos en el diagnóstico y con la percepción del derechohabiente de recibir información y vigilancia deficientes durante la hospitalización. El texto completo en inglés de este artículo está disponible en: http://ww w.insp.mx/salud/index.html

Palabras clave: quejas médicas; atención médica; factores asociados; México ing hospitalization. The English version of this paper is available at: http://www.insp.mx/salud/index.html

Key words: complains; medical care; associated factors; Mexico
$\mathrm{L}$ a interposición de quejas contra los médicos en los servicios públicos y privados de salud, los juicios por negligencia o mala práctica y el reclamo para rembolso de gastos efectuados por los pacientes inconformes con la atención recibida, han sido una práctica común en los países industrializados, y en México comienzan a observarse cada vez con mayor frecuencia. ${ }^{1-3}$

Desde la constitución de la Comisión Nacional de Arbitraje Médico (Conamed) se tiene un registro más detallado y confiable de las inconformidades que presentan los usuarios de los servicios de salud por la atención recibida en las instituciones de salud, tanto públicas como privadas. Los reportes de quejas interpuestas ante dicho organismo muestran un incremento sostenido desde 1996 hasta 2002. ${ }^{3}$

Las quejas presentadas ante la Conamed en el Distrito Federal en los años 1997 y 1998 están relacionadas principalmente con el tratamiento médico y quirúrgico, seguidas de los procedimientos de diagnóstico. La estructura y el proceso de la atención son el principal motivo de queja por parte de los usuarios hacia las instituciones de salud pública, en tanto que para las instituciones privadas el principal motivo es el resultado de la atención recibida. ${ }^{4}$

Estudios en otros países han hallado que la presencia de eventos adversos durante la atención médica, debidos o no a mala práctica, no siempre se asocian a quejas o demanda de pago contra el personal médico, y que un gran número de estas demandas son archivadas por ausencia de negligencia médica.,

La percepción que el usuario tiene sobre la calidad de la atención médica recibida es un elemento que se ha medido en sociedades con características diferentes, en donde el cumplimiento de las necesidades personales y de seguridad en la institución de salud que atiende a los derechohabientes se han asociado a la presentación de quejas por baja satisfacción., 7,8

Una buena comunicación y una adecuada relación entre el médico tratante y el paciente son elementos que en diversos estudios se han considerado funda- mentales para incrementar la satisfacción de los usuarios de los servicios de salud y disminuir la frecuencia de quejas y de procedimientos de rembolso, pago por daños o percepción de atención médica deficiente o inadecuada. ${ }^{9-11}$

No se encontraron en la literatura estudios en nuestro país que evalúen objetivamente los factores asociados a la presentación de queja ante una autoridad, administrativa o penal, por la atención médica recibida, según la percepción de los pacientes.

El objetivo del presente estudio fue identificar los factores que se asocian con la presentación de queja formal contra el personal médico en unidades hospitalarias del Instituto Mexicano del Seguro Social (IMSS), en la Delegación Estado de México Oriente, en un periodo de tres años.

\section{Material y métodos}

Se revisaron los expedientes clínicos y los formatos de queja de todos los pacientes que presentaron una queja formal contra los médicos tratantes entre enero de 1996 y enero de 1999, en los ocho hospitales generales y un hospital de ginecología y obstetricia de la Delegación Estado de México Oriente del IMSS.

Se consideraron los expedientes de aquellos casos que siguieron el trámite de queja formal a través de la Oficina Delegacional de Orientación y Quejas, las que fueron derivadas a dicha oficina por organismos oficiales como Conamed, la Comisión Nacional de Derechos Humanos (CNDH) o la Procuraduría General de la República (PGR), y cuyo motivo fuera la atención recibida por los médicos adscritos a las unidades hospitalarias del IMSS en la Delegación.

Se excluyeron los expedientes de las quejas por motivos que no fueran inherentes a las acciones puramente médicas, por ejemplo, la falta de dotación de medicamentos o material de curación, cita prolongada, quejas dirigidas contra enfermeras, trabajadores sociales, asistentes médicas u otro tipo de personal 
de salud, y también aquellos expedientes con información incompleta para su análisis.

Los expedientes fueron revisados por dos de los investigadores por medio de una lista de cotejo, con el fin de establecer los motivos reales de la queja. Cuando no hubo acuerdo al respecto, el expediente fue evaluado por un tercer revisor y en el caso de continuar con desacuerdo el expediente en cuestión se excluyó. El motivo de la queja debió estar explícito en la misma para evitar variabilidad en la interpretación entre los revisores.

Se evaluaron nueve factores establecidos en la literatura $^{3,4,12}$ asociados a la interposición de queja o demanda y cinco más que se consideraron empíricamente de importancia, debido a su frecuencia como motivo de queja y a las características de la atención que se otorga en el IMSS. Los factores evaluados fueron definidos de la siguiente manera:

- Sexo del paciente: características fenotípicas del paciente que permiten clasificarlo como femenino o masculino.

- Sexo del médico: características fenotípicas del médico tratante contra el que se interpuso la queja, que permiten clasificarlo como femenino o masculino.

- Complicación: cualquier evento adverso derivado del proceso de la atención médica y que en el formato de la queja fue anotado como complicación.

- Mala relación médico-paciente: cuando existió algún conflicto en la relación entre el médico y el paciente, o el familiar de éste, que haya sido anotado en el formato de queja.

- Deficiencia en la atención del trabajo de parto: percepción subjetiva de la derechohabiente o su familiar de que la atención durante el trabajo de parto no fuera la esperada y se estuviera en desacuerdo con la misma.

- Error diagnóstico: cuando se argumentó en el formato de queja que el diagnóstico emitido fue erróneo.

- Error terapéutico: cuando se argumentó en el formato de queja que el tratamiento recibido no fue el correcto.

- Uso de auxiliares de diagnóstico: cuando la utilización de éstos haya comprometido al médico tratante en la toma de una decisión médica, en su solicitud u omisión, o en la realización del procedimiento, como es el caso de los estudios radiológicos o endoscópicos.

- Información insuficiente: señalamiento expreso en la queja de haber recibido información insuficiente del médico tratante, según la percepción del paciente o su familiar.

- Alta voluntaria: la decisión del paciente para ser egresado por su propia voluntad de la unidad hospitalaria donde recibía atención.

- Vigilancia deficiente del paciente durante la hospitalización: inconformidad del paciente o su familiar sobre el cuidado y vigilancia que el médico ofreció durante el internamiento.

- Diferimiento de cirugía en la sala de urgencias: de acuerdo con la percepción del paciente o familiar, tiempo prolongado para realizar algún tratamiento quirúrgico durante su estancia en el servicio de urgencia.

- Revisiones vaginales múltiples preparto: desacuerdo por la revisión múltiple vía vaginal previa a la atención del parto.

- Insatisfacción con la atención recibida por el médico tratante: inconformidad explícita con la atención médica recibida, señalada en la queja por el paciente o su familiar.

El grupo control estuvo conformado por expedientes de pacientes de los mismos hospitales en los que se presentaron las quejas, pareados hasta donde fue posible por edad, sexo, diagnóstico y servicio en el que se prestó la atención médica, a razón de dos controles por cada caso.

A una muestra aleatoria de los sujetos del grupo control se les hizo una entrevista telefónica estandarizada por medio de un cuestionario elaborado ex profeso que contenía preguntas sobre los factores de riesgo estudiados, su opinión respecto a la atención recibida y la posibilidad de interponer en el futuro una queja por el servicio recibido, con el fin de excluir de este grupo a los pacientes que pudieran posteriormente presentar una queja. Las posibilidades de respuesta para el último cuestionamiento fueron a) definitivamente sí, b) probablemente sí, c) no sé, d) probablemente no, y e) definitivamente no.

Los expedientes fueron excluidos en el caso de no haber contactado a los sujetos control por la vía telefónica en un periodo de tres meses desde la atención recibida. Los expedientes de los sujetos que no contaban con teléfono fueron sustituidos por otros con los mismos criterios de inclusión y en los que no hubiera evidencia de queja formal ante la Oficina Delegacional de Información y Quejas de la Delegación, después de un año desde el momento de su atención.

Se efectuó un análisis bivariado mediante prueba de Ji cuadrado, y por medio de razón de momios se calculó el riesgo de los médicos para ser objeto de una 
queja. Utilizando el paquete estadístico SPSS 9 se realizó un modelo de regresión logística con las variables que presentaron significancia estadística en el análisis bivariado.

\section{Resultados}

El total de expedientes de queja por cualquier motivo en los nueve hospitales de la Delegación en el periodo estudiado fue de 402, y en 156 se señalaba al médico como motivo principal de la misma. Se incluyeron 130 expedientes de queja que cumplieron con los criterios de selección y que tuvieron registros completos para su análisis.

Del grupo control, conformado por 260 expedientes, se localizaron vía telefónica a 56 pacientes o sus familiares. De quienes respondieron, 52 (92.4\%), contestaron que probablemente no y definitivamente no presentarían una queja. Los otros 208 expedientes que conformaron el grupo control fueron seleccionados de los archivos clínicos de las unidades estudiadas entre aquellos que un año después de haber recibido la atención médica no evidenciaron la interposición de queja formal. Las características generales de los expedientes de derechohabientes que interpusieron queja contra los médicos del Instituto, y las de los controles, así como las especialidades donde recibieron la atención se muestran en el cuadro I.

Las proporciones de quejas por cada especialidad médica fueron: ginecobstetricia $33.1 \%$, medicina de urgencias $13.1 \%$, cirugía general $12.3 \%$, medicina interna $11.5 \%$, medicina familiar $9.2 \%$, anestesiología 3.8\% y otras $13.2 \%$.

Las quejas recibidas directamente en la Oficina Delegacional de Información y Quejas del IMSS fueron $69.2 \%$, las que se recibieron por la Conamed 23.1\%, por la CNDH $3.8 \%$, por la PGR $3.9 \%$, y a través de la dirección o de las jefaturas de servicio de los propios hospitales $3.9 \%$.

El tiempo promedio transcurrido entre la atención médica otorgada y la interposición de la queja fue de 95.1 días (rango de 9 a 450 días).

El análisis bivariado del riesgo para ser sujeto de queja por los factores mencionados se muestran en el cuadro II. Resaltan con un riesgo mayor los relacionados con la percepción de error diagnóstico, error en la terapéutica, utilización de estudios de gabinete, la decisión del paciente de ser egresado voluntariamente y la idea de una vigilancia hospitalaria deficiente por el médico.

Con las variables que tuvieron una asociación significativa se realizó un análisis de regresión logística que se muestra en el cuadro III.

\section{Cuadro I \\ Características generales de las Quejas CONTRA MÉDICOS DE HOSPITALES Y DE LOS CONTROLES. Delegación Estado de México Oriente del Instituto Mexicano del Seguro Social, 1996-1999 $\begin{array}{cc}\text { Quejas } & \text { Controles } \\ n=130 & n=260\end{array}$}

Edad en años de los pacientes Media (desviación estándar) $38.2(19.2) \quad 40.57(21.4)$

Sexo de los pacientes (\%)

\begin{tabular}{lll} 
Mujeres & $83(63.8) \quad 164(63.1)$ \\
\hline
\end{tabular}

Hombres $47(36.2) \quad 96(36.9)$

Sexo de los médicos (\%)

\begin{tabular}{lll} 
Mujeres & $34(26.5) \quad 57(21.4)$ \\
\hline
\end{tabular}

Hombres $\quad 96(73.5) \quad 203(78.6)$

Pacientes por servicio (\%)

\begin{tabular}{lrc} 
Urgencias médicas & $48(36.9)$ & $88(33.9)$ \\
\hline Medicina interna & $6(4.6)$ & $20(7.7)$ \\
\hline Cirugía general & $11(8.5)$ & $37(14.2)$ \\
Unidad toco quirúrgica & $34(26.1)$ & $77(29.6)$ \\
\hline Anestesiología & $4(3.1)$ & - \\
\hline O ftalmología & $4(3.1)$ & - \\
\hline Ginecología & $15(5.8)$ & \\
\hline O tros & $15(11.5)$ & $23(8.8)$
\end{tabular}

N ota: no hubo diferencias estadísticamente significativas entre grupos en ninguna variable

\section{Discusión}

En los últimos años, por medio de encuestas dirigidas a medir la satisfacción del derechohabiente y sus familiares, diversas instituciones, entre las que se incluye al IMSS, han dado una importancia relevante a la percepción sobre la atención médica que proporcionan. De acuerdo con datos de la Encuesta Nacional de Salud II (ENSA-II) ${ }^{13}$ la percepción sobre la calidad de la atención en el IMSS fue buena en $46.6 \%$, señalándose como los tres principales motivos de mala atención a los tiempos de espera prolongados, con $29.3 \%$, resultados de salud nulos o negativos en $10.9 \%$, y revisión y diagnósticos no realizados en $10 \%$, mientras que para otras instituciones de salud pública los resultados nulos o negativos fueron la principal causa de percepción de mala calidad con $35.8 \%$.

La encuesta de calidad y calidez del IMSS realizada en 1990 en 33 hospitales reportó que 80\% de los derechohabientes estuvieron satisfechos con la relación 


\section{Cuadro II \\ Factores asociados a la presentación de Quejas contra médicos de hospitales. Delegación Estado de México Oriente del Instituto Mexicano del Seguro Social, 1996-1999}

\begin{tabular}{lccc} 
& $p$ & $R M$ & $I C 95 \%$ \\
Sexo de los pacientes & $n$ & 1.03 & $0.66-1.06$ \\
\hline Sexo del médico & ns & 1.26 & $0.77-2.05$ \\
\hline Complicaciones & 0.001 & 2.08 & $1.50-5.46$ \\
\hline Mala relación médico-paciente & 0.009 & 2.44 & $1.22-4.88$ \\
\hline Deficiente atención del trabajo de parto & 0.014 & 3.04 & $2.64-3.51$ \\
\hline Error diagnóstico & 0.000 & 3.53 & $2.07-6.0$ \\
\hline Error terapéutico & 0.000 & 2.58 & $1.54-4.30$
\end{tabular}

Uso de auxiliares de diagnóstico

\begin{tabular}{lccr} 
Laboratorio & ns & 1.33 & $0.22-8.11$ \\
\hline Gabinete & 0.011 & 3.52 & $1.25-9.93$ \\
\hline Información insuficiente & 0.003 & 2.77 & $1.38-5.55$ \\
\hline Alta voluntaria & 0.000 & 7.72 & $2.48-23.97$ \\
\hline Vigilancia deficiente del paciente & 0.031 & 5.16 & $0.98-26.96$ \\
\hline Diferimiento de cirugía de urgencia & 0.048 & 2.30 & $0.98-5.36$ \\
\hline Multirrevisiones preparto & 0.028 & 3.34 & $1.07-10.43$ \\
\hline Insatisfacción con el médico & ns & 1.88 & $0.78-4.56$
\end{tabular}

N ota: análisis bivariado

\section{Cuadro III \\ Principales factores identificados como motivo DE QUEJA CONTRA LOS MÉDICOS DE HOSPITALES. Delegación Estado de México Oriente del Instituto Mexicano del Seguro Social, 1996-1999}

\begin{tabular}{lrrr} 
& Valor de $\mathrm{p}$ & $\mathrm{RM}$ & \multicolumn{1}{c}{ IC $95 \%$} \\
Complicaciones & 0.004 & 2.84 & $1.38-5.81$ \\
\hline Error diagnóstico & 0.000 & 3.18 & $1.77-5.69$ \\
\hline Uso de estudios de gabinete & 0.020 & 3.72 & $1.22-11.31$ \\
\hline Información insuficiente & 0.014 & 2.64 & $1.22-5.74$ \\
\hline Alta voluntaria & 0.001 & 7.25 & $2.22-23.67$ \\
\hline Falta de vigilancia & 0.007 & 19.15 & $2.25-162.3$ \\
\hline Revisiones múltiples preparto & 0.007 & 5.17 & $1.56-17.0$
\end{tabular}

N ota: análisis de regresión logística

médico paciente y $81 \%$ refirieron haber tenido una orientación suficiente y satisfactoria en la consulta externa. ${ }^{14}$

En el presente estudio se confirmaron como factores asociados a la interposición de queja contra los médicos de los hospitales a la presencia de complicaciones, error en el diagnóstico y la utilización de auxiliares de gabinete. Otros factores que se identificaron estuvieron en relación con el proceso de la atención médica, de los cuales se pueden resaltar la percepción por parte del derechohabiente o sus familiares de haber recibido una información insuficiente durante el periodo de hospitalización, y la solicitud de alta voluntaria, que fueron significativas en el modelo de regresión y que pudieran estar asociadas con una deficiente relación médico-paciente, la cual en el análisis bivariado resultó significativa.

Otros autores ${ }^{4,15}$ en nuestro país han mencionado que los factores asociados al proceso de la atención, principalmente la deficiente comunicación entre el médico y su paciente, son causa de insatisfacción que afecta la relación entre el prestador del servicio y el usuario. Otro motivo de queja lo constituyó la percepción de deficiencia en la atención durante el trabajo de parto en el cual la realización de revisiones vaginales múltiples fueron el motivo principal. Si bien en estudios previos la inconformidad y quejas de las pacientes se han asociado con los servicios, tanto de planificación familiar como de ginecología y obstetricia, ${ }^{4}$ no se ha especificado cuáles son los motivos reales de la queja. Sin embargo, en el presente trabajo se identificaron como factores asociados a las quejas la inconformidad de las derechohabientes, tanto por haber percibido una atención deficiente durante el trabajo de parto, como de haber sido objeto de revisiones vaginales múltiples.

Como otros autores lo han anotado, ${ }^{4,16,17}$ varios de los factores que en el presente estudio se asociaron a riesgo se pueden conjuntar dentro del mismo rubro de la relación médico-paciente. Estos factores de riesgo incluyen la falta de satisfacción del paciente o sus familiares con la información recibida, la percepción de una vigilancia deficiente del paciente durante su hospitalización y, como consecuencia de ellos, la solicitud por el derechohabiente de su alta voluntaria.

Hasta el momento se consideraba empíricamente que los factores asociados que fueron evaluados formaban parte de los motivos por los que un derechohabiente entablara una queja formal o demanda ante las instancias del Instituto o de organismos encargados de dirimir, administrativa o legalmente, dicha inconformidad. Los resultados obtenidos en este trabajo hacen objetiva la magnitud de los factores de riesgo por los cuales un médico puede ser motivo de queja formal o demanda en su contra.

No fueron objetivos de este trabajo hacer juicios de valor sobre la actuación del personal médico, ni establecer si las quejas de las que fueron objeto estaban fundamentadas, ya que la queja por la demanda de un servicio está sustentada, básicamente, en la per- 
cepción que el paciente o sus familiares tienen por el servicio recibido. Tampoco se trató de establecer si el número de quejas contra los médicos del IMSS fue elevado respecto a otras instituciones, públicas o privadas, que brindan atención médica. Aunque el Informe Anual de Labores de $2002^{18}$ de la Conamed reporta un número de inconformidades mayor para el IMSS, debe considerarse que el número de unidades de atención médica del Instituto y el número de servicios otorgados es mayor que el de otras instituciones.

Se requiere de más estudios que evalúen en forma prospectiva los factores identificados, con el fin de poder incidir en el mejoramiento, tanto de los aspectos técnicos como de los relacionados con el proceso de la atención, en los que se incluyen las relaciones humanas entre los médicos del Instituto y los derechohabientes.

\section{Conclusiones}

Los motivos de queja contra los médicos especialistas que laboran en las unidades hospitalarias del IMSS en la Delegación Oriente del Estado de México se asocian principalmente con aspectos técnicos en el diagnóstico, y con la percepción del derechohabiente de recibir información y vigilancia insuficientes durante la hospitalización. Factores todos ellos relacionados con el proceso de la atención médica que requieren de la implantación de estrategias específicas para su mejoramiento, con el fin de facilitar la comunicación y la relación médico-paciente, lo cual pudiera reflejarse en un descenso en el número de quejas.

\section{Referencias}

1. Brennan TA, Leape LL, Laird N M, Hebert L, Localio AR, Lawthers AG. Incidence of adverse events and negligence in hospitalized patients. Results of the Harvard Medical Practice Study I. N Eng J Med 1991;324:370-376.
2. Fernández-Varela-Mejía H. Síntesis ejecutiva del informe de actividades. D écimo novena sesión ordinaria del Consejo. Rev Conamed 2000;4(15):47-51.

3. Comisión N acional de Arbitraje Médico. Información estadística Institucional Conamed 2002. Disponible en: www.conamed.gob.mx/ atendidos.htm. Consultado el 12 de diciembre de 2003.

4.Valdez-Salgado R, Molina-Leza J, Solís-Torres C. A prender de lo sucedido.Análisis de las quejas presentadas ante las Comisión $\mathrm{N}$ acional de Arbitraje Médico. Salud Publica Mex 2001;43:444-454.

5. Localio AR, Lawyhers AG, Brennan TA, Laird N M, Hebert LE, Peterson LM. Relation between malpractice claims and adverse events due to negligence. Results of the Harvard Medical Practice Study III. N Engl J Med 1991;325:245-251.

6. Brennan TA, Sox CM, Burstin HR. Relation between negligent adverse events and the outcomes of medical-malpractice litigation. N Engl J Med 1996;335:1963-1967.

7. Rees-Lewis J. Patients views on quality care in general practice: Literature review. Soc Sci Med 1994;39:655-670.

8. Hall JA, D orman MC.W hat patients like about their medical care and how often they asked. A meta analysis of satisfaction literature. Soc Sci Med 1998;27:935-939.

9. Kravitz RL, C allahan EJ, Paternini D,Antonius D, D unham M, Lewis CE. Prevalence and sources of patients' unmet expectations for care.Ann Intern Med 1996:125:730-737.

10. 0 wen C. Formal complaints against general practitioners:A study of 1000 cases. Br J Gen Pract 1991;41:113:-115.

11. Levinson W, Gorawara-Bhat R, Dueck R, Egener B, Kao A, Kerr C. Resolving disagreements in the patient-physician relationship:Tools for improving communication in managed care. JAMA 1999;282:1477-1483. 12. Halperin EC. G rievances against physicians. Eleven years' experience of a medical society grievances committee. West J Med 2000;173:235238.

13. Ramírez-Sánchez TJ, N ájera-A guilar P, N igenda-López G . Percepción de la calidad de la atención de los servicios de salud en México: perspectiva de los usuarios. Salud Publica Mex 1998;40(1):1-12. 14. Aguirre-G as H. Evaluación de la atención médica. Expectativas de pacientes y trabajadores. Salud Publica Mex 1990;32(2):170-180.

15. Tena-Tamayo C . La demanda como efecto de la mala comunicación médico-paciente. Rev Conamed 2002;7(3):20-23.

16. Hernández-G amboa L. Relación médico-paciente y la calidad de la atención médica. Rev Conamed 2001;20:25-29.

17. Tena-Tamayo C, Ruelas-Barajas JM, Sánchez-G onzález AE, RiveraCisneros AE, Moctezuma-Barragán G, Manuell-Lee GR. Derechos de los pacientes en México. Rev Med Inst Mex Seguro Soc 2002;40:523-529. 18. Comisión N acional de Arbitraje Médico. Informe Anual de Labores 2002 Conamed 30D. Disponible en: www.conamed.gob.mx/ difusion.html. Consultado el 12 de diciembre de 2003. 